Nouvelles perspectives en sciences sociales

Revue internationale de systémique complexe et d'études relationnelles

\title{
Le système interactionnel : connexions sémantiques et contextique relationnelle
}

\section{Albin Wagener}

Volume 7, numéro 2, mai 2012

Sur le thème de la modélisation

URI : https://id.erudit.org/iderudit/1013055ar

DOI : https://doi.org/10.7202/1013055ar

Aller au sommaire du numéro

Éditeur(s)

Prise de parole

ISSN

1712-8307 (imprimé)

1918-7475 (numérique)

Découvrir la revue

Citer cet article

Wagener, A. (2012). Le système interactionnel : connexions sémantiques et contextique relationnelle. Nouvelles perspectives en sciences sociales, 7(2),

67-104. https://doi.org/10.7202/1013055ar
Résumé de l'article

Si la systémique permet de mettre en exergue la complexité des interactions humaines (Meunier 2003), elle a aussi pour but d'en préciser les modalités de régulations et les éléments permettant à ces interactions d'émerger et de se maintenir (Varela et al. 1993). En reliant cette systémique interactionnelle à des principes hérités du connexionnisme (Bechtel et Abrahamsen 1993, Marcus 2001), nous affirmons qu'il devient alors possible de mettre l'accent sur l'importance du contexte dans les interactions et sur la manière dont les individus réagissent en fonction de noeuds sémantiques, dans la mesure où ces noeuds se retrouvent notamment activés lors de processus d'identification nécessaires pour l'individu ou le groupe (Burke et Stets 2009). Le présent travail théorique explore ces possibilités, en lien avec la prise en compte de la complexité des relations humaines (Lerbet-Séréni 1994, Wagener 2010). 


\title{
Le système interactionnel : connexions sémantiques et contextique relationnelle
}

\author{
Albin Wagener \\ Université catholique de l'Ouest \\ LALIC/PPI, PRES L'UNAM \\ UCO - IPLV
}

\section{Introduction}

$A$ près avoir observé des situations de malentendus interA culturels ${ }^{1}$, nous avons été en mesure d'en déduire que ceux-ci correspondaient à des critères propres à tout système vivant $^{2}$. Toutefois, nous avons constaté que, bien que les êtres humains disposent de moyens relativement sophistiqués pour faire vivre et évoluer leurs relations et leur façon de communiquer, l'essence même de ces interactions repose sur des fondements nécessaires à toute existence du vivant. Nous proposons d'utiliser les paradigmes systémiques, pragmatique et connexionniste afin

$1 \quad$ Albin Wagener, "On Intercultural Disagreement: Interaction and Inertia ", dans Reyes Gomez Moron, Manuel Padilla Cruz, Lucia Fernandez Amaya et Maria de la O Hernandez Lopez (dir.), Pragmatics Applied to Language Teaching and Learning, Newcastle upon Tyne, Cambridge Scholars Publishing, 2009, p. 256-279.

2 Albin Wagener, "Cooperative Disagreement: Towards a Definition of Discordant Interactions ", dans Iwona Witczak-Plisiecka (dir.), Pragmatic Perspectives on Language and Linguistics, vol. 1, Speech Actions in Theory and Applied Studies, Newcastle upon Tyne, Cambridge Scholars Publishing, 2010, p. 171-197. 
de représenter les nuances et les subtilités des rapports humains. Un modèle systémique des interactions humaines ou système interactionnel repose avant tout sur la souplesse et l'adaptabilité de ses paramètres. Le but principal du présent article est de proposer une hypothèse de modèle théorique, cohérent et compréhensif des dynamiques interactionnelles, pour une restitution plus fidèle de la complexité des interactions humaines, notamment lorsque celles-ci subissent des situations de déséquilibre conflictuel.

\section{Principes élémentaires systémiques}

Afin de mettre progressivement en place le modèle du système interactionnel, il convient d'abord de spécifier les éléments fondamentaux que nous souhaitons emprunter à la systémique. Tout d'abord, il est important de noter que les mécanismes de base du système interactionnel fonctionnent sur le principe de cohérence :

La cohérence implique tous les aspects paradoxaux liés à l'autonomie : la fermeture sur soi, une certaine confusion entre ce qui agit et ce qui est agi, la présence du tout dans la partie faisant pendant à la présence de la partie dans le tout. (...) Le fait que l'on puisse caractériser la cohérence en disant que chaque partie se comporte comme si elle était informée de l'état de l'ensemble implique que chaque interaction locale s'effectue en fonction de l'état global qu'elle cherche à maintenir ${ }^{3}$.

En d'autres termes, il est possible de comprendre une relation ou un conflit de façon globale en replaçant les éléments systémiques dans leur contexte d'émergence, dans la mesure où tous fonctionnent véritablement en interaction les uns avec les autres, soutenant ainsi le principe de cohérence : les notions même de cause et d'effet doivent alors laisser place à une co-construction de sens constante, changeante et soumise à un certain nombre de conditionnements environnementaux, qu'ils soient internes ou externes au système, ainsi que de règles particulières. Les premiers conditionnements concernent les premiers éléments du système interactionnel, à savoir les interactants eux-mêmes.

3 Jean-Pierre Meunier, Approches systémiques de la communication, Bruxelles, De Boeck, 2003, p. 59-60. 
Ceux-ci investissent différemment le système selon un certain nombre de conditionnements d'ordre psychologique, sociologique, éducatif, culturel, biologique, génétique ou physiologique, entre autres. Lorsque deux individus se rencontrent, ils portent en eux le fruit de ces différentes influences, sans compter l'historique de la relation en cours : il est évident que la relation qui lie les individus prendra une place importante dans le déroulement de l'interaction - que l'on se connaisse depuis longtemps ou non, qu'il s'agisse d'une relation professionnelle, amicale, amoureuse ou familiale, ces différentes formes de rapports nous apportent des données capitales pour l'analyse du système interactionnel.

Historiquement, la théorie générale des systèmes pose les bases de la systémique contemporaine et prend sa source au cœur de divers courants scientifiques, en s'illustrant notamment par les influences qu'elle doit à l'idée de totalité cybernétique. Ce courant va notamment générer une théorie des systèmes qui s'éloigne de l'analogie entre le fonctionnement de l'homme et celui de la machine, posant ainsi les bases de la systémique ${ }^{4}$ : tout système est constitué d'éléments qui interagissent entre eux. En fonction de cette définition, nous pouvons proposer le schéma suivant :

$4 \quad$ Ludwig Von Bertalanffy, Théorie générale des systèmes, Paris, Dunod, 1973, p. 23-30. 
Figure 1

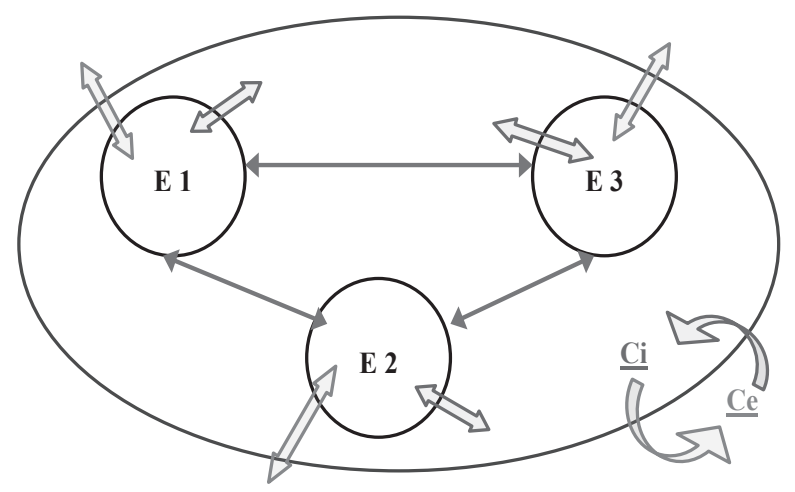

Le schéma simplifié ci-dessus (que nous complexifierons par la suite), représente le système en tant que tout, dont l'organisation est apparentée à celle d'une cellule, incluant à la fois les éléments $(1,2$ et 3 ) et les relations qui les lient. II inclue également le contexte intérieur (Ci) qui présente l'intégralité des paramètres existants au sein même du système, ainsi que le contexte extérieur $(\mathrm{Ce})$, dans lequel est plongé le système tout entier et avec lequel il entretient des relations constantes.

Le modèle d'étude systémique est capable d'étudier différents objets en les incluant dans l'intégralité du système ils font partie (ou " tout »), évitant ainsi un isolationnisme analytique qui ne rend pas compte de la complexité d'un phénomène. Si l'on imagine trois individus qui se rencontrent, par exemple, ceux-ci noueront entre eux des relations qui n'auront de cesse d'agir tout au long de la durée de l'interaction. De surcroît, les empreintes laissées par ces relations perdurent au-delà de l'interaction ellemême. Cependant, le système en lui-même est loin de n'être constitué que d'individus en interaction; en effet, il renferme également des notions de temps et d'espace, qui peuvent être à la fois intérieures ou extérieures au système, ainsi que des conditions circonstancielles qui influeront fortement sur les communications des interlocuteurs. Ainsi, nous pouvons d'ores et déjà avancer le postulat suivant : dans toute interaction, les individus sont plongés dans un "bain » contextuel et agissent en fonction de lois systémiques qui influent sur leurs relations, tout en permettant de les comprendre et de les expliquer. Afin de comprendre le fonctionnement élémentaire d'un système, il faut 
être familiarisé avec trois phénomènes communs à tout système vivant :

- l'émergence, soit " les qualités ou propriétés d'un système qui présentent un caractère de nouveauté par rapport aux qualités ou propriétés des composants considérés isolément ou agencés différemment dans un autre type de système ${ }^{5}$;

- les conditionnements organisationnels qui régissent les complémentarités entre les éléments du système et son ordre de manière générale, évitant ainsi l'éclatement du système et autorisant également une certaine forme de redondance, notion que nous explorerons plus loin;

- la dyade complémentarité/antagonisme, qui représente le système comme un ensemble d'éléments qui agissent de façon conjointe pour l'émergence et le maintien homéostasique, tout en maintenant leurs antagonismes dans une logique de différenciation.

Cependant, l'énumération des éléments et des phénomènes systémiques serait vaine si nous n'examinions pas le but ontologique de tout système vivant, à savoir l'homéostasie du système lui-même par la transmission d'informations.

\section{De la circulation d'information}

Le rôle de l'information est déterminant dans tout système quel qu'il soit : sa circulation seule garantit l'existence même du système, dans la mesure où " une information acquise permet de restaurer dans le système une différence, c'est-à-dire un état plus improbable, c'est-à-dire encore, de l'ordre ${ }^{6}$ ». En ce sens, il n'y a ni bonne, ni mauvaise circulation d'informations; la communication vit, évolue et passe d'un élément à un autre du système en fonction de certaines règles et altérations. Dans un système interactionnel, la manière que les individus auront d'interpréter les informations créera précisément de la nouveauté et de la

5 Edgar Morin, La Méthode, Tome 1, La nature de la nature, Paris, Seuil, 1977, p. 106.

6 Jean-Pierre Meunier, Approches systémiques de la communication, Bruxelles, De Boeck, 2003, p. 21. 
différence dans l'expression de leurs points de vue. C'est également en ce sens que pourront alors émerger des déséquilibres interactionnels; il est alors important de définir le concept de redondance, qui permet de comprendre la pertinence des habitudes sociales, linguistiques, psychologiques ou culturelles des individus, qui vont reproduire un certain nombre d'occurrences communicationnelles, comme par exemple les rituels de salutations, afin de pouvoir créer un certain confort cognitif dans leurs rapports. Si nous devions à chaque fois réinventer nos manières d'interagir et nos codes de communication, il serait fort difficile de pouvoir construire des relations durables. La redondance, « constituée par les répétitions internes au message qui le rendent plus prévisible » joue un rôle capital dans la transmission d'informations, et donc dans l'existence même de tout système; elle conditionne le décryptage les messages en se référant à un certain nombres de repères, de codes ou de comportements déjà connus des individus. En d'autres termes, la redondance organise le fonctionnement du système et de ses éléments, en minimisant les dépenses d'énergie, dans un souci d'efficacité. En systémique, cette économie d'énergie est appelée " néguentropie ${ }^{8}$ " et maximise la transmission d'informations avec le minimum d'énergie possible. En ce sens, il est important de garder en mémoire le rapport entre néguentropie et information ${ }^{9}$, dans la mesure où l'information redondante constitue une meilleure organisation du système et donc une valorisation de la néguentropie - ou économie d'énergie. Une telle déduction est notamment soutenue par l'existence de codes culturels ou sociaux qui organisent les sociétés, grâce à l'économie d'énergie procurée par la redondance de ces codes dans la communication. Cependant, il est également nécessaire d'apporter de la variation et de la nouveauté dans la circulation d'informations.

$\mathrm{Au}$ cœur des interactions humaines, de tels principes sont importants et soulignent le fait que nous sommes des créatures

Ibid., p. 17.

8 Joël de Rosnay, Le Macroscope, Paris, Seuil, 1975, p. 172.

9 Olivier Costa de Beauregard, Le Second Principe de la science du temps, Paris, Seuil, 1963, p. 71-72. 
pétries d'habitudes. En tant qu'êtres vivants, nous suivons un mode de fonctionnement caractéristique de notre condition, qui se retrouve aussi bien dans la plus petite cellule que dans les organisations mondiales importantes comme l'ONU : la transmission d'information maintient la vie, et pour ce faire, un certain nombre de règles sont à respecter. Tout système est sujet à autorégulation qui conditionne ses ajustements aux éventuelles modifications internes et externes. Lorsque des modifications apparaissent, elles peuvent évoluer selon trois stades, définis par Ilya Prigogine et Isabelle Stengers ${ }^{10}$ :

- la fluctuation, qui agit sur le système comme une perception par les éléments d'une tentative de modification : il s'agirait pour les individus de ressentir certains comportements comme inconfortables ou menaçants, par exemple;

- la nucléation, qui structure la modification en cours au sein du système en lui accordant un emplacement propre : dans le cas du système interactionnel, il s'agirait alors pour les individus de discuter au sujet des comportements perçus comme menaçants;

- l'amplification, qui permet finalement à la modification de prendre de l'importance au cour du système : par exemple, un conflit interactionnel ouvert, peut aller jusqu'à la violence verbale et physique.

L'utilisation de ces trois notions facilite l'étude de l'émergence des conflits interactionnels de façon pertinente, en tentant de délimiter ses trois étapes d'expression. Pour empêcher la nucléation d'un désaccord dans un système interactionnel, la condition sine qua non reste la rapidité de la transmission d'informations entre les individus, au sujet des éléments qui font émerger le point de désaccord.

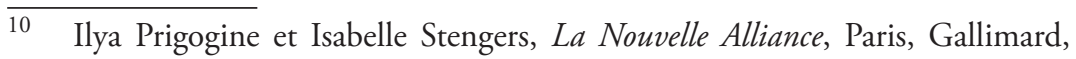
1979 , p. 169. 
Tableau 1

\begin{tabular}{|c|c|c|c|c|}
\hline & Interaction & Communication & Fluctuations & $\begin{array}{c}\text { Probabilité de } \\
\text { nucléation }\end{array}$ \\
\hline $\begin{array}{l}\text { Stabilité de } \\
\text { l'état du } \\
\text { système }\end{array}$ & \multirow{2}{*}{ Diversifiée } & Rapide & Insignifiantes & faible \\
\hline $\begin{array}{l}\text { Possibilité } \\
\text { d'instabilité } \\
\text { du système }\end{array}$ & & Lente & Dangereuses & élevée \\
\hline
\end{tabular}

Ce tableau permet de définir l'état de stabilité d'un système, et par extension, d'un système conversationnel.

Moins les éléments du système communiquent entre eux, plus le risque d'amplification devient réel; nous pouvons observer cette situation lorsque deux individus choisissent de ne plus se parler, laissant ainsi le conflit s'installer de façon durable dans des proportions impropres à la communication. Dans une autre mesure, l'éclatement de bulles financières en 2008 a mis à jour l'existence d'une crise boursière importante, qui a émergé au moment où des points de nucléation plus ou moins cachés dans le système ont explosé et dilué les éléments du système dans un phénomène d'amplification accentuée. Le modèle même du système doit parfois passer par des phases d'amplification afin de pouvoir être profondément modifié et répondre à de nouveaux besoins. En définitive, soit le système rentre dans un schéma qui le stabilise par redondance, mais de frustrer une partie des besoins de certains de ses éléments, soit le système subit une modification en brisant le modèle duquel il fait partie en amplifiant les fluctuations qui l'habitent. En outre, les individus, en tant qu'éléments du système interactionnel, se plient également à ces processus pour maintenir le système en place; en situation professionnelle, lorsqu'un individu refuse de se plaindre à propos d'une situation de harcèlement subi, il assure ainsi la pérennité du système et le protège dans un besoin de relatif confort émotionnel et cognitif. En ce sens, les individus sont soumis à des éléments contextuels variés et variables. 


\section{Contextes et complexité contextique}

En tant que système dans le système, l'individu en interaction est perpétuellement soumis à un certain nombre de contraintes plus ou moins contradictoires. En produisant une nouvelle perspective, les individus se positionnent de manière différente et interconnectent les éléments de savoir en leur possession d'une nouvelle manière : ils perçoivent les choses sous un nouvel angle. Nous nous référons ici déjà à l'approche connexionniste que nous approfondirons plus loin dans ce travail en insistant sur les propriétés de nouvelles interconnexions: "les théories et les modèles (...) s'appuient sur une armée de composants analogues à des neurones, simples, inintelligents, qui, quand ils sont connectés de façon adéquate, présentent des propriétés globales intéressantes ${ }^{11}{ }^{1}$. L'acquisition de points de vue différenciés garantit l'évolution dans la connaissance : plus un individu aborde des points de vue différents, plus son champ de connaissances est susceptible de s'étendre et de se complexifier, même si cette intégration se fait déjà d'un point de vue particulier. Pour reconnaître un nouveau point de vue, il s'agira donc de l'intégrer en reprogrammant les interconnexions existant entre les éléments, de façon plus ou moins consciente. Finalement, il est important de souligner le fait que les systèmes interactionnels ne sont pas hermétiques au monde dans lequel ils évoluent, d'où leur grande complexité et le besoin de pouvoir trouver une façon adéquate de la modéliser; de plus, chaque individu implique dans l'interaction les expériences qu'il a vécues avant la mise en place du système. Ces différentes fenêtres sur l'univers extérieur qualifient le système interactionnel comme un système ouvert, entretenant avec l'univers et abritant en lui-même des interactions continues. En conséquence, la représentation du système interactionnel devient justement complexe :

$\overline{11}$ Fransico Varela, Eleanor Rosch et Evan Thompson, L'Inscription corporelle de l'esprit. Sciences cognitives et expérience humaine, Paris, Seuil, 1993, p. 134. 
Figure 2

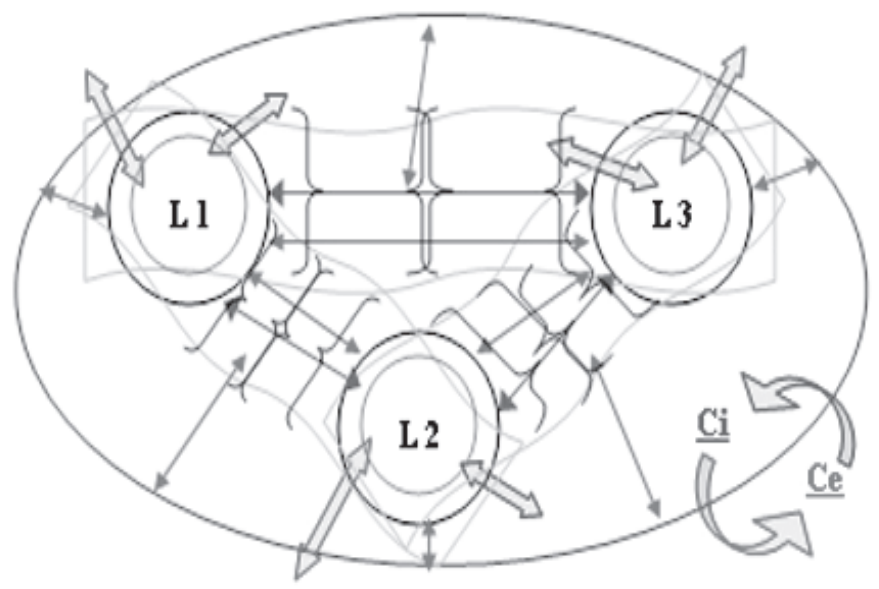

Ce schéma représente trois interlocuteurs en interaction dans un système donné. Chaque locuteur (L1, L2 et L3) dispose d'un filtre modélisateur (représenté à l'intérieur du locuteur) et entretient avec le système, avec ses interlocuteurs et avec l'univers extérieur des relations permanentes, qu'elles soient en rapport ou non avec son filtre modélisateur (ce qui justifie les deux types de flèches parallèles). Chaque filtre modélisateur représente une perspective différente, car propre à chaque interactant et fonction de son expérience propre, et s'inscrit directement dans le concept de redondance entretenu par le système interactionnel et les individus. Le système entretient également des relations avec l'univers qui lui est extérieur. A ces éléments viennent s'ajouter les relations entretenues avec les contextes intérieur et extérieur au système, qui s'échangent également des informations entre eux. Les phénomènes de redondance (vagues encadrant les interationcs) permettent d'éviter les interférences causés par les bruits (ici représenté par les accolades), qu'ils se produisent au niveau du filtre modélisateur de l'interactant ou non, ou même au beau milieu de la transmission du message. Pour des raisons de lisibilité, nous avons choisi de ne pas faire figurer sur le schéma le fait que les notions de redondance et de bruit s'appliquent tout autant entre le système lui-même et l'un de ses éléments.

Toutefois, le modèle systémique, malgré ses nombreux avantages, présente un point faible remarquable : il est en effet difficile d'y faire figurer les influences nombreuses et prégnantes exercées par les différents contextes auxquels les interactants sont confrontés. L'importance des contextes ne doit pas être minorée, puisque ce sont eux qui vont modeler une grande partie de nos interactions. Sans la variété des contextes (interaction dans un ascenseur, un train, une chambre à coucher ou un dîner d'affaires), il est 
absolument impossible de pouvoir analyser les rapports entre les éléments du système interactionnel. Il est alors impérieux de remettre cette notion au centre même de nos préoccupations interactionnistes, précisément parce que nous avons besoin de contextualiser les informations que nous recevons, souvent de façon non consciente, afin de pouvoir faire sens et agir en conséquence :

Dans toute interaction humaine, il y a des gens qui sont en train de faire quelque chose ensemble, à un moment donné, dans un lieu donné. Pour comprendre de manière contextique comment fonctionne cette interaction, il importe d'observer, non tellement le contenu de ce qui est dit ou fait, mais la façon dont les gens opèrent pour agir. Ce faisant, on peut éventuellement repérer qui dirige qui, qui manipule qui, et peutêtre qui manipule l'observateur. Comme il y a ce que les gens font, ce qu'ils disent qu'ils font et ce qu'ils croient qu'ils font, on peut aussi agir en faisant croire qu'on fait autre chose ${ }^{12}$.

Si nous suivons la théorie de Paul Castella, il vaudrait mieux parler de contextique plutôt que de systémique, dans la mesure où le contexte doit d'abord être entendu comme un processus en perpétuelle évolution. Nous définissons donc le contexte comme processus de contextualisation développé par Paul Castella, dans la mesure où « les séquences, les épisodes, les scénarios personnels, les modèles culturels, les habitudes de comportement, sont des domaines de signification susceptibles de devenir contextes dans un processus de contextualisation. En hiérarchisant ces différents domaines, on les met en jeu d'une certaine façon : on donne du sens. Ce que nous appelons la réalité est résultat de cette contextualisation ${ }^{13}$ ". Si nous souscrivons à cette définition, nous souhaitons la compléter en signalant que le contexte, en tant que processus évolutif, n'est pas forcément effectué en toute conscience par les interactants. D'une certaine manière, la contextique est un outil d'observation du déroulement d'une interaction en fonction du contexte et de son développement global. Dans cette optique, les expressions et actions des interactants, en prise avec différents éléments contextuels, deviennent elles-mêmes des

12 Paul Castella, La Différence en plus, Paris, L'Harmattan, 2005, p. 270.

13 Ibid., p. 143. 
émergences du contexte, des occurrences qui suivent la logique d'un tout. Cependant, tout en évitant un paradigme par trop déterministe, nous estimons que le système interactionnel se développe à la manière d'un organisme vivant; il passe par un certain nombre de choix et accepte certaines modifications internes et externes. Les activités des interactants font partie de cet organisme que nous nourrissons par nos relations, nos perceptions et nos interprétations.

Connaître le contexte, ce n'est pas simplement obtenir une vision globale de l'évolution de l'organisme interactionnel : c'est également comprendre les points d'ancrage et de mouvement qui garantissent l'existence du système. Les tours de parole, les fines transitions que nous effectuons lorsque nous changeons de sujet, la façon dont les environnements influencent notre physiologie ou notre façon de comprendre les informations à notre disposition, tout cela fait partie d'une base essentielle au vivant. En d'autres termes, la contextique s'appuierait sur la prise en compte d'un contexte général qui alimenterait de manière pressante les liens sémiologiques entre les éléments du système, afin de pouvoir en saisir les principes fondamentaux et les mécanismes qui soutiennent son autorégulation. Selon nous, cette vision théorique apporte une manière originale d'étudier les interactions humaines, en mettant l'accent sur le fait que leurs aspects psychologiques, sociologiques ou culturels n'en sont que des couleurs différentes, des déclinaisons variables parfois mises en scène par l'observateur lui-même. Une analyse contextique des interactions, ainsi qu'une étude sémiotique des tensions qui les parcourent et les constituent, mettent en lumière certains principes utiles à la compréhension, la résolution ou même la prévention de conflits interactionnels. Selon nous, pour représenter un tel modèle, il convient de mettre en place une image complexe, tridimensionnelle et évolutive dans le temps pour avoir une vue d'ensemble des subtilités et des nuances qui agitent les interactions humaines. Ce faisant, nous aurions ainsi accès à l'ensemble des informations contextuelles que le cerveau interprète comme pertinentes, ponctuellement ou durablement, afin de comprendre les com- 
portements des individus et l'évolution de leurs relations. Le but d'une telle entreprise, soutenu par la problématique du présent article, est de mieux représenter, et ainsi de mieux comprendre la manière dont certains rapports humains peuvent parfois se détériorer et éventuellement de pouvoir prévoir ou prévenir l'apparition de certains déséquilibres, autrement que de manière empirique, avec un maximum d'éléments à notre disposition. Le point d'entrée des modifications liées aux déséquilibres de ces rapports humains est représenté par les perceptions émotionnelles des individus.

\section{4. Émotions et motivations en conflit}

Afin d'approfondir l'approche contextique des systèmes interactionnels, il est important de comprendre la manière dont les individus peuvent être amenés à construire le sens à travers leurs relations, précisément pour mieux comprendre les points d'articulation sémiologiques précédemment évoqués. Au cours des interactions, les individus ont conscience d'accéder à certaines informations, et l'interprétation de celles-ci pourra modifier leur façon de percevoir leur environnement et leur interlocuteur. Une précédente étude ${ }^{14}$ a tenté de montrer à quel point les émotions deviennent une valeur cardinale lorsqu'il s'agit de comprendre l'émergence des conflits interactionnels, dans une perspective interculturelle. Cette étude reposait sur une analyse de discours rapportés par des individus après que ceux-ci aient vécu un conflit : leurs productions langagières donnaient l'impression de traces émotionnelles distinctes après le passage d'une situation interactionnelle inconfortable. Dans cette perspective, nous souhaitons préciser la définition du conflit, empruntée aux importants travaux de Michel Monroy et Anne Fournier, que nous employons tout au long du présent article:

[Le conflit est] à la fois choisi et subi. Alors que chacun des adversaires le nourrit de ses convictions et de ses initiatives, il a en même temps le

14 Albin Wagener, "L'Émotion dans l'émergence du désaccord interculturel ", dans Béatrice Pothier (dir.), Langue, langage et interactions culturelles, Paris, L'Harmattan, 2009, p. 133-153. 
sentiment de se limiter à subir et à réagir aux initiatives de l'adversaire, ou à la fatalité de la situation. (...) Le conflit dissocie, désagrège, déconstruit le système en même temps qu'il le reconstruit autrement ${ }^{15}$.

Il n'est pas aisé de savoir à quel moment d'un déséquilibre systémique émerge un conflit; selon nous, il y a conflit à partir du moment où il y a modification importante de la configuration initiale du système. En soi, peu importe de savoir si le conflit est accidentel, nécessaire ou inhérent au système interactionnel : si les conditions sont réunies, il peut émerger et modifier le système en profondeur. Dans ce cadre, ce sont les émotions qui jouent un rôle primordial, puisqu'elles agissent comme des " états motivationnels ${ }^{16}$ " et nous ouvrent le choix de certaines actions ou expressions plutôt que d'autres. En d'autres termes, si un individu est submergé par la gêne, ses actions seront précisément conditionnées par cet état émotionnel : il pourra rougir, avoir les mains qui tremblent, bégayer et avoir du mal à organiser ses idées. Nous ne souhaitons pas ici affirmer que nous sommes les esclaves de nos émotions : il appartient à chacun de pouvoir les canaliser, le cas échéant. Cependant, quelle que soit l'émotion ressentie, celle-ci aura une influence sur nos activités ultérieures, puisque ces mêmes émotions s'inscrivent dans des contextes particuliers. Imaginons par exemple qu'un conducteur, en pleine possession de ses moyens, se mette à ressentir un état émotionnel de stress lorsqu'il se retrouve arrêté par la maréchaussée pour un simple contrôle de papiers du véhicule. Il conviendra de prendre en compte les éléments suivants :

- le comportement altéré du conducteur pourra être ressenti comme suspect par l'officier de police et introduire un nouvel élément contextuel;

- l'émotion ressentie par le conducteur s'inspire d'un vécu personnel, basé soit sur de mauvaises expériences anté-

15 Michel Monroy et Anne Fournier, Figures du conflit, Paris, Presses universitaires de France, 1998, p. 10.

16 Nico Frijda, "Passions : l'émotion comme motivation ", dans Jean-Marc Colletta et Anna Tcherkassof (dir.), Les Émotions. Cognition, langage et développement, Sprimont, Mardaga, 2003, p. 15. 
rieures (rapports conflictuels avec des officiers de police), soit sur une empreinte psychologique de la crainte de l'autorité;

- l'état émotionnel pourra être jugulé ou accentué en fonction des nouveaux éléments contextuels qui apparaîtront au cour du système interactionnel (apparence, intonation et vocabulaire de l'officier de police, par exemple).

Comme nous le voyons, l'état émotionnel d'un individu pourra avoir des répercussions sur l'ensemble du système. Les émotions sont nécessaires et leur présence est permanente : elles sont chargées de nous communiquer des choix de réactions ou d'actions possibles en fonction de l'interprétation d'éléments extérieurs. Elles participent à la constitution et l'évolution de nos processus identitaires ${ }^{17}$ et déterminent les bases de nos opérations cognitives en associant certaines opérations à des impressions agréables ou désagréables ${ }^{18}$. De notre point de vue, les émotions peuvent être définies comme des réponses aux influences de l'environnement, induisant un certain nombre d'actions possibles dans une situation précise. Les émotions sont également liées à la mémoire ${ }^{19}$ et sont stockées afin de pouvoir faire face à un événement similaire dans de nouvelles situations, dans une perspective de prévisibilité et d'économie d'énergie. Nous pouvons ainsi en déduire les processus suivants :

- un nouveau contexte $\mathrm{C} 1$ fait émerger de nouvelles émotions E1, émotions qui participent à leur tour à la modification de ce contexte : $\mathbf{C 1} \rightarrow(\mathbf{E} \mathbf{1})$;

- ces émotions E1 induisent de nouvelles réactions R1 qui vont renforcer les émotions dans une optique de circularité (E1) $\leftarrow \rightarrow($ R1)

17 Peter Burke et Jan Stets, Identity Theory, Oxford, Oxford University Press, 2009, p. 174.

18 Gilles Kirouac, Cognition et émotions, Coimbra, Imprensa da Universidade de Coimbra, 2004.

19 Bernard Rimé, Marie-Pascale Noël et Pierre Philippot, «Épisode émotionnel, réminiscences mentales et réminiscences sociales ", Les Cahiers internationaux de psychologie sociale, $\mathrm{n}^{\circ} 11,1991$, p. 93-104. 
- parallèlement à cela, ces mêmes émotions E1 sont stockées et restent en mémoire $\mathbf{M}$ chez l'individu : $\mathbf{M}=(\mathbf{E} 1)$;

- les réactions qu'elles induisent peuvent alors servir à nouveau lorsqu'un contexte similaire $\mathrm{C1}$ ' au contexte originel émerge derechef : si C1', alors $\mathbf{M} \rightarrow(\mathbf{E} 1+\mathbf{R} 1)$.

Ces différents éléments peuvent être représentés grâce au schéma suivant :

Figure 3

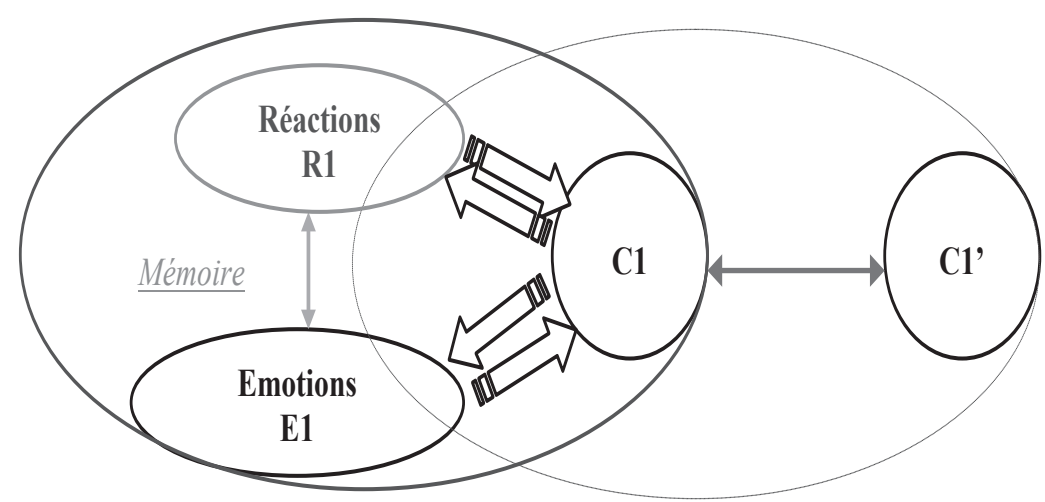

Ce schéma représente ici l'expérience mémorisée d'un contexte C1, en relation directe avec un ensemble d'émotions E1 et de relations R1, qui émergent ellesmêmes dans un processus d'ordre circulaire; cette expérience mémorisée est rappelée lors d'un contexte $\mathrm{C1}^{\prime}$ ' considéré comme similaire par l'individu.

En tout état de cause, il se peut qu'un contexte interprété comme semblable à un autre contexte précédemment expérimenté en soit parfois suffisamment éloigné pour que de nouvelles émotions et de nouvelles réactions dussent en émerger. D’un point de vue métacognitif ${ }^{20}$, les contextes émergent par un certain nombre d'associations internes qui utilisent les expériences précédemment acquises comme des "filets de sécurité " dans notre rapport au monde. Quelles que soient les situations, que nous voyons un chat traverser la route pendant que nous conduisons, que nous élaborions une stratégie de séduction ou que nous passions un examen, nos émotions vont influencer nos

20 Benjamin Baars, In the Theater of Consciousness. The Workspace of the Mind, Oxford, Oxford University Press, 1997, p. 125. 
choix cognitifs et nos références à des contextes précédents, comme le précise Nico Frijda :

Les émotions influencent les pensées de diverses façons, suscitant ou réfrénant l'élaboration cognitive, créant et fixant des croyances, déterminant l'acceptation ou le rejet d'informations. Toutes ces influences se comprennent en grande partie comme des actions servant à faire face à la situation émotionnelle ou à se servir des opportunités rencontrées ${ }^{21}$.

Mais que se passe-t-il lorsque ces émotions interagissent avec nos comportements cognitifs lors de conflits interactionnels? Que se passe-t-il lorsqu'une relation dérape ou qu'un geste est mal interprété? Parfois, un regard, un geste ou une intonation peuvent être mal perçues, précisément parce que nous ne sommes pas habitués à les recevoir dans un contexte particulier. C'est ce que nous appelons parfois de l'impolitesse, tout simplement parce que les expressions de notre interlocuteur ne cadrent pas avec nos habitudes cognitives et menacent ainsi notre confort émotionnel. Il s'agit ici d'un processus correspondant à un principe de pertinence : ce qui ne rentre pas dans le cadre cognitif et émotionnel habituel est soit ignoré (il ne peut alors pas être sémantisé à proprement parler), soit se trouve confiné hors cadre au rang d'élément perturbateur. À ce titre, nous estimons que tout conflit émerge lorsqu'un comportement est interprété comme menaçant ou agressant par un individu; sans cette interprétation, les relations gardent une certaine forme d'harmonie ${ }^{22}$.

\section{De la menace perçue aux mécanismes de défense}

Dans ces contextes conflictuels, les individus agissent comment s'ils se défendaient contre une agression supposée : soit il s'agit de maitriser cette situation d'inconfort tout à fait inédite en canalisant nos émotion ${ }^{23}$, ce qui enrichit alors notre perspective relationnelle, soit il s'agit d'exclure les nouveaux éléments rencontrés. Il est toutefois nécessaire de souligner le fait que nous

\footnotetext{
21 Nico Frijda, op. cit., p. 24.

22 Albin Wagener, "Cooperative Disagreement... », op. cit.

23 Isabelle Paulhan et Marc Bourgeois, Stress et coping. Les stratégies d'ajustement à l'adversité, Paris, Presses universitaires de France, 1995.
} 
construisons nous-mêmes les interprétations qui nous mènent à nous sentir agressés dans une situation donnée. Plusieurs mécanismes de défense peuvent alors émerger ${ }^{24}$, quelle que soit la relation qui nous lie à notre interlocuteur. Cette dystasie survient parce que nous nous ancrons dans un système interactionnel via des nerfs émotionnels, qui sont autant de liens que nous entretenons avec les points d'articulation sémantique d'un système. Nos liens au système interactionnels sont nécessaires à la transmission d'information et sont investis par des univers sémantiques structurés. Nous sommes ainsi en capacité de produire, parfois malgré nous, des "blends expérientiels " lorsque la situation l'exige, faisant ainsi fi d'une cohérence mentale précise :

Il s'agit de mélanger des percepts et des « représentations » - la sortie ou le résultat consistant en une " perception associée à une représentation " bien plus qu'en une "représentation de perception " (...). La construction du blend expérientiel semble donc primer, dans l'expérience, sur le maintien d'une cohérence représentationnelle ${ }^{25}$.

Les blends expérientiels sont une manière de faire émerger des activités cognitives, des réactions ou des comportements nouveaux par l'interaction d'éléments cognitifs et émotionnels précédents et actuels; ils autorisent à la fois la redondance du système tout comme le risque d'émergence d'un conflit, en constituant, selon les situations, un mécanisme de défense. Ces mécanismes, selon Hanna Malewska-Peyre, constituent " "ensemble de manœuvres” pour éviter l'angoisse ou la dévalorisation. On pourrait parfois utiliser un autre terme comme "mécanismes défensifs", mais il s'agit parfois non seulement de réactions inconscientes de défense, mais également de réponses conscientes actives $^{26} »$.

24 Serban Ionescu, Claude Lhote et Marie-Madeleine Jacquet, Les Mécanismes de défense, Paris, Armand Colin, 2005.

25 Antoine Auchlin, "Compétence discursive et co-occurrence d'affects : "blends expérientiels" ou (con)fusion d'émotions ", dans Jean-Marc Colletta et Anna Tcherkassof, Les Émotions. Cognition, langage et développement, Sprimont, Mardaga, 2003, p. 145.

26 Hanna Malewska-Peyre, "Problèmes d'identité des adolescents enfants de migrants et travail social ", dans Carmel Camilleri et Margalit CohenEmerique, Chocs de cultures : concepts et enjeux pratiques de l'interculturel, Paris, 
Figure 4

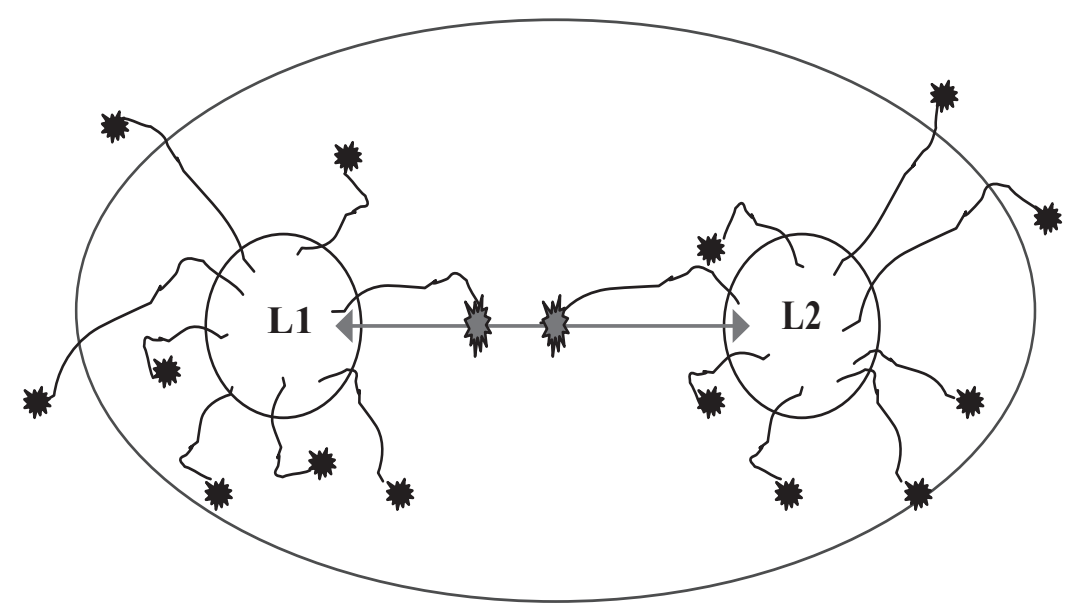

Le schéma simplifié ci-dessus représente deux participants à un système interactionnel à travers la métaphore des neurones et de leurs dendrites, ancrés dans le système (y compris dans la relation liant les individus, en vert) et à l'extérieur de celui-ci à travers une variété de points de sémantisation.

En outre, afin d'embrasser la complexité des règles auxquelles sont soumis les interactants, il est important d'intégrer à cette vision systémique les apports de la pragmatique concernant la manière dont les individus gèrent les buts et investissements sémantiques dans les relations.

\section{Principes pragmatiques et compréhension de la complexité}

Les règles établies par les travaux en pragmatique peuvent s'appliquer à toute forme de communication quelle qu'elle soit. L'un des principes les plus essentiels, souvent discuté et retravaillé par les chercheurs de ce domaine, est le principe de coopération :

Nos échanges de parole ne se réduisent pas normalement à une suite de remarques décousues et ils ne seraient pas rationnels, si tel était le cas. Ils sont de manière caractéristique, jusqu'à un certain point au moins, le résultat d'efforts de coopération; et chaque participant reconnait jusqu’à un certain point dans ces échanges un but commun ou (...) au moins une direction acceptés réciproquement. Ce but ou cette direction peuvent être fixés dès le départ, ou bien ils peuvent apparâtre au cours

L'Harmattan, 1989, p. 117-134, p. 123. 
de l'échange; ils peuvent être relativement bien définis ou assez vagues pour laisser une latitude considérable aux participants ${ }^{27}$.

Selon nous, l'existence de tels buts ne signifie pas nécessairement que les interactants aient besoin de se mettre d'accord concernant leur interaction. D'une certaine manière, les individus ne peuvent s'empêcher d'atteindre un but commun, tant que la communication est maintenue, quelle que soit la nature de leur relation ou la teneur de la conversation en cours. Il est important de remarquer que, même dans les situations de conflit ${ }^{28}$, les individus agissent encore en partageant la même vision de la relation avec un but commun, à savoir l'affrontement; ceux-ci se comportement comme s'ils étaient sur un ring de boxe, acceptant de facto l'existence d'un certain nombre de règles qui encadrent la joute dans un espace défini. Dans ce cas de figure, même si l'accord sur le but n'est pas nécessairement conscient, maintient tout de même l'interaction. D'un point de vue systémique, la " ceinture coopérative » apporte donc une assise supplémentaire au maintien du système interactionnel.

Dans un second temps, le principe de négociabilité ${ }^{29}$ souligne un élément important des interactions : celles-ci sont sans cesse négociées, aussi bien dans leur contenu que dans leur forme pour Werner Kallmeyer, il n'y a pas de coopération sans négociation constante entre les individus, démontrant ainsi la base de l'interaction. Parfois, cette négociation est automatisée et acceptée de façon moins conscientisée par les interactants, notamment lorsque deux individus se rapprochent, passant d'une relation amicale à une relation amoureuse, par un jeu subtil de partage de nouvelles sémantisations. Parfois également, elle est consciente, comme dans le passage du vouvoiement au tutoiement. Lors de l'analyse des interactions, il s'agit d'être attentif à la façon dont certains traits sémantiques saillants peuvent être négociés par les interlocuteurs, que ceux-ci en soient ou non conscients. D'une

27 Paul Grice, «Logique et conversation », Communications, n³0, 1979, p. 60.

28 Albin Wagener, "Cooperative Disagreement... ", op. cit.

29 Werner Kallmeyer, "L'Analyse de l'action dans la conversation », dans Pierre Bange, L'Analyse des interactions verbales. La dame de Caluire. Une consultation, Bern, Peter Lang, 1987, p. 189-251. 
autre façon, le principe de négociabilité montre également que les individus peuvent ou non utiliser les influences qui les animent pour bouleverser l'équilibre du système interactionnel.

En dernier lieu, le principe de réciprocité, développé par David Lewis $^{30}$ souligne quant à lui un autre état de fait : lorsque la coopération fonctionne et qu'elle s'exprime à travers la négociation de certains traits pertinents, ceux-ci ont alors un impact réciproque sur les individus eux-mêmes. D'une certaine manière, le principe de réciprocité s'appuie sur un partage d'informations au cour de l'interaction, puisque les individus ajusteront leurs comportements en fonction de l'interprétation de ces informations. La réciprocité fonctionne également dans les relations discordantes, puisqu'il s'agit alors d'une divergence d'interprétation.

Ces trois principes pragmatiques sous-tendent les bases systémiques des rapports humains : nous coopérons, nous négocions et nous ressentons sans cesse les effets réciproques de nos choix respectifs. Selon nous, ces trois points pragmatiques sont mis en mouvement par la politesse, en tant que force motrice importante des relations humaines ${ }^{31}$ : elle utilise un certain ensemble de comportements et de codes préférentiels auxquels les individus se réferent afin de pouvoir faciliter les opérations cognitives et relationnelles exigées par le besoin d'harmonie sociale. Si nous rattachons la politesse aux éléments pragmatiques, celle-ci représente l'application emblématique des principes de coopération, de négociabilité et de réciprocité, qui tous maintiennent la relation au cœur du système interactionnel.

30 David Lewis, Convention. A Philosophical Study, Harvard, Harvard University Press, 1969.

31 Pierre Bange, Analyse conversationnelle et théorie de l'action, Paris, Hatier/ Didier, 1992; Catherine Kerbrat-Orecchioni, La conversation, Paris, Seuil, 1996. 
Figure 5

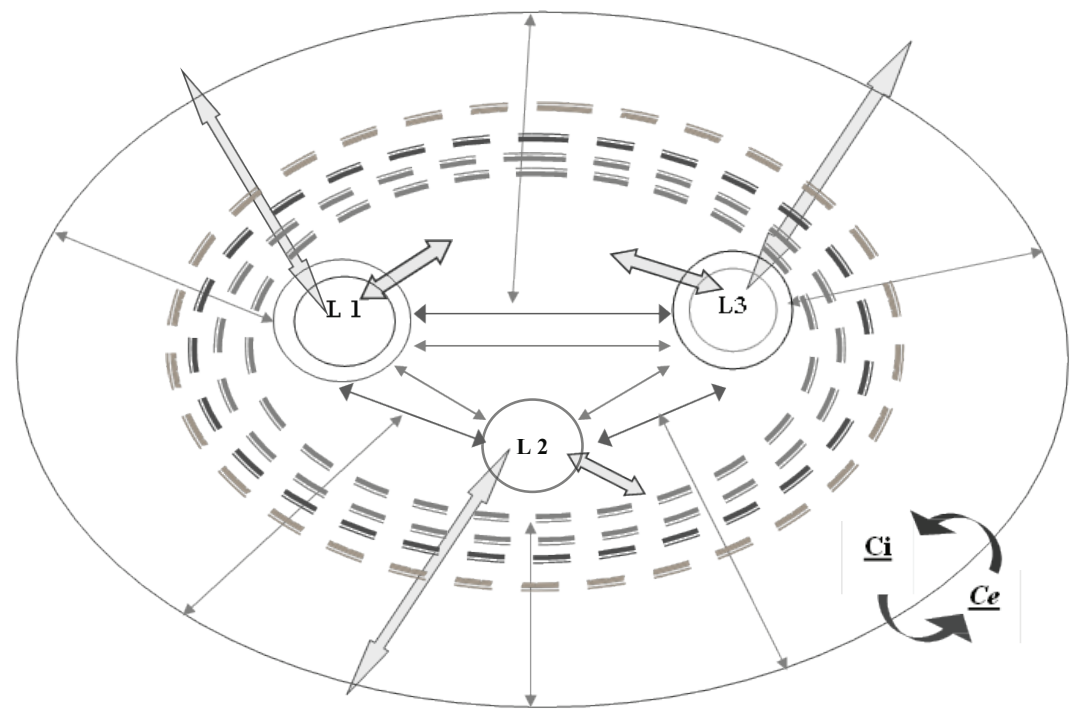

Nous pouvons résumer ces quatre principes grâce au schéma ci-dessus : ils constituent ici quatre ceintures qui permettent d'encadrer les échanges entre les trois interlocuteurs, à savoir la politesse, la réciprocité, la négociabilité et la coopération (dans un ordre d'intérieur vers l'extérieur). Ces ceintures orbitales permettent, en raison de leur éloignement autour des individus, de pouvoir définir leur degré d'implication au sein du système interactionnels : plus les cercles concentriques sont éloignés, plus ils sont génériques et participent au fonctionnement basique de l'interaction. Ainsi, comme nous l'avons expliqué précédemment, la politesse se retrouve plus proche des interlocuteurs en ce qu'elle constitue un réservoir d'outils afin de ménager leurs faces, alors que le principe de coopération apparaît bien plus éloigné, puisqu'il est un principe fondamental au sein des processus de communication et d'interaction.

\section{La relation au cour du système interactionnel}

Il est nécessaire de souligner que les liens existant entre les éléments du système jouent un rôle capital. Dans ce sens, les relations entretenues par les individus constituent un point important du contexte interactionnel : celles-ci sont investies et sémantisées de façon différente suivant les personnes. D'un point de vue systémique, la relation participe de façon intégrale du fonctionnement du tout et émerge en fonction des rapports historiques des individus, comme le précise Frédérique Lerbet-Séréni :

[Il y a] émergence de (...) la " création du couple ", espace tiers, interfaciel, investi par l'un et l'autre élément. Les frontières de ce système 
avec l'environnement sont variées dès lors que le couple s'emploie à développer un noyau commun en contact, lui aussi, avec l'extérieur. La création et l'enrichissement de ce troisième terme à partir des projets communs aux deux membres du couple orientent le système et contribuent à le finaliser ${ }^{32}$.

De cette façon, la relation, en tant que "troisième terme ", devient pratiquement un élément à part entière, une sorte d'hologramme émergeant des événements communicationnels interindividuels et animant les interactants à travers leurs rapports. La relation suit ainsi un schéma propre susceptible de mener les individus vers un conflit, tant ceux-ci vont l'investir de manière importante. La relation maintient le lien communicationnel et donne du sens nouveau à certains points d'ancrage dont ont besoin les individus. Quelle que soit la relation, il est vraisemblable que chaque interactant en aura une vision différente et relativement personnelle : les conditionnements psychologiques, sociologiques, éducatifs ou familiaux donneront une couleur particulière au filtre qui permet à chacun de percevoir la relation dans laquelle il est engagé. Il nous semble alors important de détailler les points suivants :

- dans tout système interactionnel, les individus investissent leur relation et la font évoluer comme élément à part entière du système;

- chaque interactant possède une perspective personnelle de cette relation;

- un déséquilibre émerge lors d'une confrontation des interprétations de la relation;

- l'émergence de ce déséquilibre survient à cause d'un élément communicationnel perçu comme un acte menaçant ou potentiellement agressant par l'un des interlocuteurs.

D'après ces différentes informations, nous estimons alors que l'équation suivante est applicable à un système interactionnel, lorsque celui-ci engage deux individus : $1+1=4$. De fait, puisque les visions de la relation deviennent des éléments à part

32 Frédérique Lerbet-Séréni, La Relation duale, Paris, L’Harmattan, 1994, p. 46. 
entière de l'interaction, il est de fait que chaque individu interviendra dans le système en ayant sa propre perception du lien existant. Ces interprétations de la relation s’interpénètrent pour laisser émerger une version commune de la relation, mais chaque interprétation dispose d'un espace propre.

Figure 6

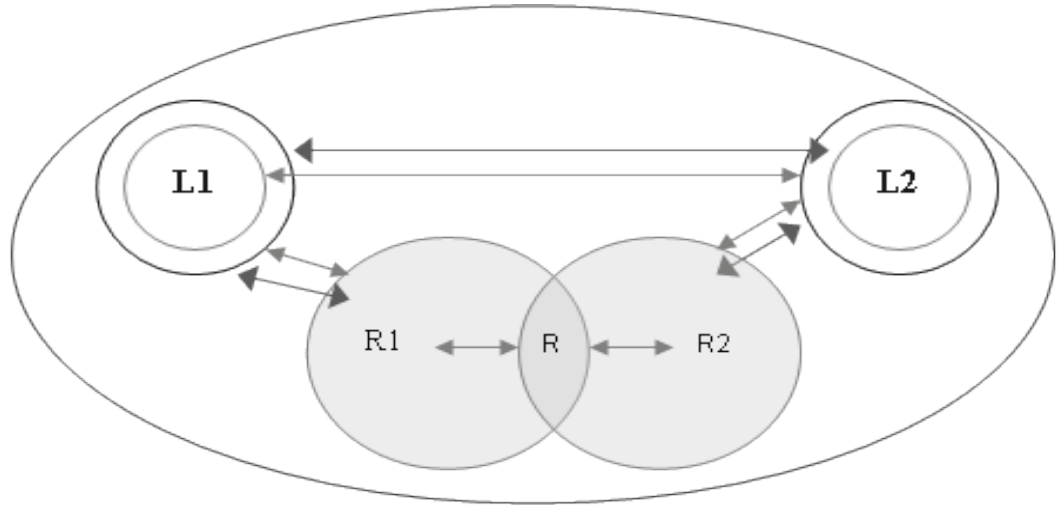

Ces deux représentations de la relation, soit $\mathbf{R} 1$ et $\mathbf{R 2}$, alimentent et conditionnent la relation $\mathbf{R}$ qui a cours entre les locuteurs en tant que partenaires interactionnels. Les deux types de flèches représentent soit les rapports maintenus entre les différents éléments du système, soit entre leurs filtres modélisateurs.

Une telle représentation de la relation interindividuelle complexifie encore le modèle des interactions humaines que nous tentons de mettre en place. Le système se trouve enrichi par un nouvel élément représentant deux nouvelles entités distinctes, et reste également soumis aux mêmes lois systémiques et principes pragmatiques. L'important est de comprendre les rapports et les articulations entre ces différents éléments et en fonction des règles analysées, afin de proposer un modèle intégratif et ouvert des interactions humaines. C'est pour cela que nous souhaitons nous inspirer de l'approche connexionniste et de son principe élémentaire : l'architecture par réseaux. 


\section{De l'émergence par réseaux de sous-systèmes}

Afin de compléter notre modèle de système interactionnel, il convient d'apporter des éléments essentiels issus des sciences cognitives afin de mieux embrasser les nuances précisées par l'approche connexionniste; la notion d'émergence, prend ici un sens fondamental et évince toute forme de localisation dans les tâches cognitives. Ainsi, celles-ci "semblent être effectuées de manière optimale par des systèmes consistant en un grand nombre de composants simples qui, quand ils sont connectés selon des règles appropriées, donnent lieu à un comportement global correspondant à la tâche désirée ${ }^{33}$ ". D'une certaine façon, l'hypothèse connexionniste postule l'élément suivant : un certain nombre de systèmes et de sous-systèmes sont soumis à des contraintes et effectuent des actions qui, mises en relation, participent à l'émergence d'un nouvel état ou d'une nouvelle action - ce qui est également le cas pour le système interactionnel. L'ensemble de ces opérations peuvent constituer la forme d'enaction dont parlent Francisco Varela, Eleanor Rosch et Evan Thompson, mais explique également la conscience humaine comme forme d'émergence ultime d'une architecture de réseaux et de sous-réseaux ${ }^{34}$. Ainsi, la mise en relation des architectures de réseaux et de systèmes représente l'émergence des rapports humains. A l'intérieur d'un système interactionnel, par exemple, plusieurs sous-systèmes peuvent être répertoriés :

- les individus eux-mêmes constituent des sous-systèmes à part entière;

- le contexte interne au système est considéré comme un sous-système;

- le contexte externe au système est lui aussi un sous-système important;

- la relation qui lie les interactants représente également un sous-système.

33 Francisco Varela, Eleanor Rosch et Evan Thompson, op. cit., p. 34.

34 Daniel Dennett, La Conscience expliquée, Paris, Odile Jacob, 1993. 
Ces éléments tissent progressivement l'émergence du système interactionnel et son maintien en tant qu'unité intégrale ouverte, notamment en utilisant la métaphore des systèmes cybernétiques précisée par Bernard Laks :

Les systèmes cybernétiques sont en effet ouverts sur leur environnement, duquel ils reçoivent les impulsions initiales, mais la sortie du système peut également être réentrante de sorte que le système fait aussi partie de l'environnement qu'il modifie. De telles boucles de rétroaction peuvent avoir des effets de stabilisation homéostatique particulièrement complexes. (...) Cet état d'équilibre, ou relaxation du système, optimise les contraintes contradictoires qui pèsent sur le système ${ }^{35}$.

En d'autres termes, afin d'exploiter correctement les travaux de Bernard Laks, ces données doivent être mises en exergue afin de décrire un système d'après le modèle connexionniste :

- la notion d'impulsion, qui représente les communications essentielles autour des points d'ancrage sémantiques (dans la mesure où ils garantissent l'interprétation d'un sens pertinent);

- l'interpénétration entre système et environnement exosystémique, qui représente de manière précise la nécessité d'une approche globale considérant des unités comme des macrosystèmes ou des microsystèmes;

- la complexité comme mode de fonctionnement du système, qui tempère la déduction de règles élémentaires qui orienterait l'analyse vers un réductionnisme dangereux;

- la notion de contradiction, dans la mesure où plusieurs sous-systèmes ou sous-réseaux peuvent nourrir des tendances paradoxales voire opposées, tout en participant à l'émergence d'un état ou d'une activité qui les dépassent tout en les mobilisant.

La contradiction ne doit pas être évacuée comme une difficulté à résoudre, mais doit être intégrée comme une condition quasiessentielle du bon fonctionnement d'un système connexionniste. Ainsi, les paradoxes ne doivent pas nécessairement être résolus,

35 Bernard Laks, Langage et cognition, Paris, Hermès, 1996. 
mais participent à l'émergence d'un tout précisément parce qu'ils existent : un système est ainsi en capacité d'intégrer à la fois un élément et son contraire sans que son homéostasie soit menacée. D'une certaine manière, cette intégration compréhensive de la contradiction remet en question le modèle causaliste, au sein duquel une action entraînerait une réaction. L'approche connexionniste n'est pas basée sur ce modèle, puisqu'elle utilise la conceptualisation de l'émergence comme interconnexions d'architectures de sous-réseaux, comme si les interactions humaines étaient représentées comme des volcans soumis à un certain nombre de forces contradictoires dont les actions individuelles ne sont elles-mêmes que des émergences. Que se passe-til lorsqu'un conflit émerge dans un système interactionnel? Le modèle causaliste soulignerait l'origine du conflit (ou élément zéro) afin de lui en attribuer la responsabilité. Nous estimons pourtant que, lorsqu'un conflit éclate entre deux individus, la responsabilité n'entre pas nécessairement en compte, dans la mesure où chaque individu participe de l'émergence de ce conflit. Il demeure ainsi important d'explorer les interactions comme des ensembles complexes soumis à des conditionnements particuliers, tout comme en météorologie ou en géophysique, par exemple. Les sciences humaines comme l'anthropologie ou la sociologie pourraient alors puiser dans les modèles hérités des neurosciences afin de construire des objets d'étude et des outils d'analyse pertinents et réalistes, tout en prenant en compte la façon qu'on les individus de pouvoir construire du sens à partir des données disponibles dans leur environnement.

\section{Nœuds sémantiques et herméneutique interactionnelle}

Afin d'obtenir une compréhension plus précise des mécanismes d'émergence au sein des systèmes interactionnels, nous estimons que le modèle causaliste doit pouvoir être remplacé par une approche par émergence de sens, notamment à travers le concept de "nœud sémantique " que nous souhaitons introduire ici. Dans ce sens, nous nous inspirons de l'approche connexionniste, qui 
évoque l'étude nodale : il est alors important de recourir au paradigme neuroherméneutique, défini par Stephen Reyna :

The neurohermeneutic system works through an interpretive hierarchy utilizing cultural memories of past realities to represent present realities in ways that form desires about future realities. Because neurohermeneutics "runs" on culture, it may be said to be a cultural neurohermeneutics. Cultural neurohermeneutics, then, is a golden bow that looses arrows of desire; and culture is part of the archer's prowess which helps guide those arrows to their target ${ }^{36}$.

La théorie de Stephen Reyna prend un point de vue résolument singulier concernant l'approche anthropologique et culturelle et propose d'étudier précisément les nœuds de connexion en se basant sur le fonctionnement neuronal, pour pouvoir obtenir une approche plus globale mais également plus complexe des choix sociaux et culturels des individus; ces nœuds de connexion fonctionnent comme autant de points de rencontre à partir desquels le sens se forme pour les interactants, afin que ceux-ci puissent interpréter et donner forme aux éléments à l'intérieur et à l'extérieur du système. Nous proposons d'appeler " nœuds sémantiques » ces points de rencontre et de connexion, dans la mesure où ils constituent autant d'indices capables d'apporter une compréhension significative des systèmes interactionnels étudiés. Si nous devions représenter un schéma interactionnel capable d'intégrer ces nouveaux éléments, il faudrait un schéma tridimensionnel susceptible de rendre compte du temps et de l'espace couverts par les individus eux-mêmes, rendant également compte de leurs expériences et de leur façon de sémantiser ces nœuds. Ici encore, une telle construction demanderait un travail considérable puisqu'elle se positionnerait à la

36 Stephen Reyna, Connections. Brain, Mind, and Culture in Social Anthropology, London, Routledge, 2002, p. 114-115; « Le système neuroherméneutique fonctionne selon une hiérarchie interprétative qui utilise les mémoires culturelles des réalités passées, afin de représenter les réalités du présent de façon à ce que celles-ci permettent l'émergence de désirs à propos des réalités à venir. Comme la neuroherméneutique fonctionne à partir de la culture, nous pouvons parler de neuroherméneutique culturelle. La neuroherméneutique culturelle devient alors un arc d'or qui envoie des flèches de désir; et la culture représente une partie de la prouesse l'art de l'archer, quant à sa capacité à capable de guider les flèches vers leur but " (notre traduction). 
croisée interdisciplinaire d'un certain nombre de champs de recherche. En effet, si nous souhaitons modéliser le système interactionnel, c'est précisément pour apporter un éclairage supplémentaire concernant la sociabilité de l'espèce humaine en termes de fondamentaux de transmission d'information. Cette démarche ontologique s'appuie sur les bases essentielles à tout système vivant pour pouvoir expliquer un certain nombre de comportements et de codes sociaux ou culturels humains, en intégrant l'émergence, la mise en place, la sémantisation et l'effectivité de ces comportements et de ces codes. Il s'agit ainsi d'embrasser les propriétés générales de l'être humain en tant que vivant interagissant, afin de cerner les enjeux de nos relations, notamment lorsque celles-ci s'expriment par le biais de désaccords ou de conflits basés sur des affrontements représentationnels et des interprétations de certaines occurrences communicationnelles comme des perceptions d'agression ou de menaces particulières. La nécessité d'une théorie synthétique des interactions humaines passe par l'étude de la complexité afin de comprendre la sophistication des stratégies singulières utilisées par notre espèce :

The notion of a core interaction engine driving human social life makes eminently good sense (...). It is not easy to isolate the critical features of such an ability, because they range from the abstract mental simulations of Schelling mirror worlds, to the concrete problems of binding across multimodal signals, or the processes generating striking crosscultural parallels across procedures for person reference. But the effort has to be worth it. Progress promises the key to understanding human evolution, and it offers to shed light on human ontogeny, higher level social processes, and the limitations of a mentality forged in face-to-face contact in the present world of nation states, superhuman agglomerations endowed by us with personal attributes they mostly do not have ${ }^{37}$.

37 Nick Enfield et Stephen Levinson, "On the Human "Interaction Engine” ", dans Nick Enfield et Stephen Levinson (dir.), Roots of Human Sociality, New York, Berg, 2006, p. 39-69; "Le concept d'un mécanisme de base des interactions, qui guide la vie sociale humaine, parait éminemment pertinent (...). Il n'est pas facile d'isoler les composantes culturelles d'une telle capacité, dans la mesure où celles-ci vont de simulations mentales abstraites comme les "miroirs" de Schelling, aux problèmes concrets de la reliance de signaux multimodaux, en passant par des processus générant d'impressionnants parallèles interculturels à travers des procédures de référence personnelle. Mais 
L'essentiel pour un modèle complexe des interactions humaines à vocation ontologique est ainsi de proposer une herméneutique basée sur les connexions et les relations entre les individus et la manière dont ceux-ci interprètent et articulent le sens selon un certain nombre de nœuds sémantiques : le tout s'opère en rapport étroit avec un contexte d'émergence. Il s'agit alors de cerner la dynamique de transmission d'information à travers ce modèle afin de rendre compte de l'infinie complexité des relations humaines et de leurs différentes déclinaisons, tout en tentant de définir un certain nombre de processus dynamiques récurrents. Bien entendu, si la complexité donne parfois l'image d'un tout dépourvu de règles, pris dans un cycle permanent et inconstant, l'espèce humaine dispose en revanche de limites biologiques, cérébrales et sociales telles qu'un certain nombre de comportements et d'expressions sont d'ores et déjà codées, tout comme le sont certaines manières d'interpréter et de produire du sens. De fait, chaque individu au sein du système interactionnel a accès à un réseau d'informations, composé lui-même de divers sousréseaux. Même lorsque l'information est partielle, l'être humain puise dans sa mémoire et son expérience pour compléter l'information par analogie avec des situations rencontrées précédemment, et ce grâce à cinq éléments délimités par les travaux de William Bechtel et Adele Abrahamsen que nous souhaitons ici reprendre et résumer ${ }^{38}$ :

- la métaphore neuronale, qui utilise les modèles en réseau et le traitement de l'information en parallèle, en liant les architectures locales et générales dans un souci d'émergence;

- la souplesse du modèle, qui préfère utiliser le terme de

le jeu en vaut la chandelle. Le progrès promet les clés de la compréhension de l'évolution humaine et offre la possibilité de porter un nouveau regard sur l'ontogénétique humaine, les processus sociaux plus généraux, ainsi que les limitations des mentalités forgées en contact direct, dans un monde actuel d'États-nations et d'agglomérations surhumaines, auxquels nous attribuons des qualités personnelles qu'ils n'ont, pour la plupart, jamais eu " (notre traduction).

38 William Bechtel et Adele Abrahamsen, Le Connexionnisme et l'esprit. Introduction au traitement parallèle par réseaux, Paris, La Découverte, 1993, p. 65-74. 
connexions nodales plutôt que d'éléments causalistes linéaires, remettant par là-même en cause l'existence de règles prédéfinies dans les interactions et assurant une analyse plus réaliste de l'adaptation;

- la " dégradation gracieuse " qui intègre également la notion de fluctuation systémique à l'ensemble de l'architecture interconnectée, tout en précisant que la disparition ou l'altération d'un élément n'inferre en rien dans l'ensemble de l'interaction à réaliser, mais qu'une certaine masse critique d'informations nucléées doit être atteinte;

- la mémoire adressable par le contenu, qui repère un certain nombre d'indices et d'éléments pour avoir accès à un tout recherché;

- enfin, la capacité de pouvoir intégrer l'expérience et d'en apprendre de nouveaux éléments transforme le système interactionnel en un ensemble mouvant et susceptible de se conformer à de nouvelles contraintes contextuelles afin de garantir sa propre pérennité.

La relation représente le lieu névralgique d'expression et d'homéostasie du système interactionnel. Celle-ci influence en effet le comportement des individus de façon importante et devient à la fois le réceptacle et le transmetteur d'un certain nombre d'informations et d'interprétations émanant des interactants. La relation constitue en quelque sorte le noyau du système (si l'on se réfere à la métaphore cellulaire) et organise le redéploiement et le traitement de bon nombre d'informations. Les individus gardent un rôle actif dans l'établissement et l'équilibrage de la relation et de ses représentations, puisque c'est par le biais de leurs propres points d'ancrage et nœuds sémantiques que ces représentations relationnelles peuvent avoir cours. Toutefois, les individus n'entrent pas directement en contact au sein du système, mais établissent l'interaction par le biais de la relation, qui garde alors un rôle de tiers pour que nous puissions comprendre son importance comme centre névralgique au niveau de la réception de l'émission d'information. D'une certaine manière, 
la relation "baigne " au cour d'une sorte de " cytoplasme " contextuel, que celui-ci soit interne ou externe au système, tout entier vecteur d'informations plus ou moins pertinentes. La relation est également alimentée par les représentations que s'en font les individus, et les représentations sont les seules interfaces garantissant la communication entre interactants et relation. En tant qu'éléments changeants et influencés par un certain nombre de conditionnements, les individus sont quant à eux reliés au monde par des nœuds sémantiques et produisent ainsi des interprétations conditionnant la communication et la transmission d'information. Le tout est ceinturé par les quatre principes pragmatiques précédemment délimités, représentés par le schéma ci-dessous.

Figure 7

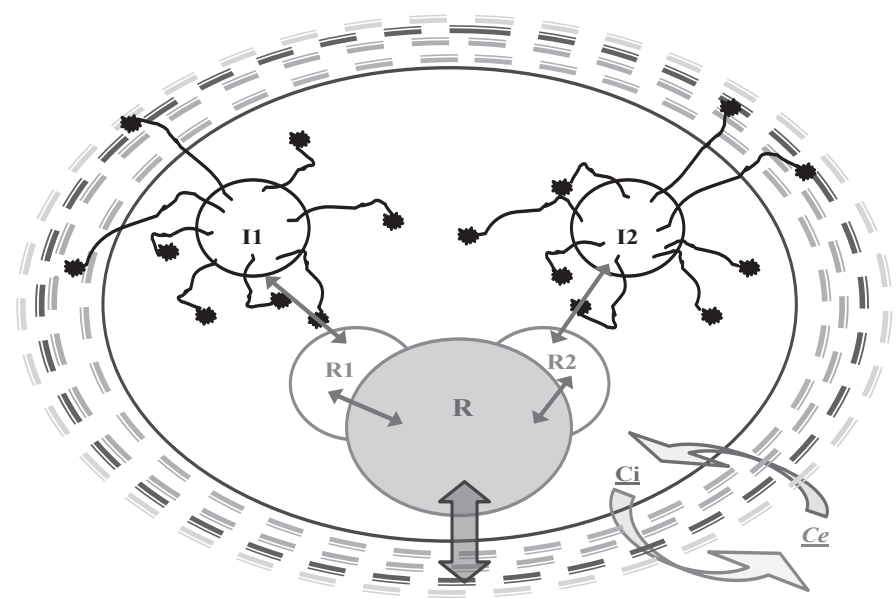

La relation $\mathbf{R}$ apparaît nettement comme le noyau central du système interactionnel. Elle se retrouve alimentée par les deux représentations $\mathrm{R} 1$ et R2, elles-mêmes nourries par les interprétations des individus I1 et I2, qui font circuler de l'information au cœur du système, basée sur les nœuds sémantiques auxquels sont reliés les interactants. $R 1$ et $R 2$ alimentent et conditionnent la relation $\mathbf{R}$ qui a cours entre les locuteurs en tant que partenaires interactionnels; de surcroît, c'est bien la relation qui entretient un rapport étroit avec les quatre principes pragmatiques schématisés à nouveau par quatre ceintures : la politesse, la réciprocité, la négociabilité et la coopération (toujours par ordre d'intérieur vers l'extérieur). Les deux participants systémiques se retrouvent liés au système (voire noués au système), qui se retrouve lui-même dans un environnement comprenant à la fois un contexte interne et un contexte externe qui restent intrinsèquement liés. 
Ce modèle est susceptible d'approfondir le fonctionnement des interactions et de cerner les zones de fluctuation lorsqu'il s'agit d'étudier les conflits interindividuels. Bien évidemment, une telle schématisation induit une forme de simplification à visée à la fois pédagogique et scientifique, mais elle reste résolument insuffisante pour représenter l'inhérente dynamique de ce système.

\section{Perspectives d'application écologiques du modèle complexe de système interactionnel}

Afin de mieux comprendre les interactions humaines, de nouvelles pistes de réflexion s'imposent : la première est notamment de pouvoir suivre la façon dont les individus activent et gèrent les nœuds sémantiques auxquels ils sont attachés afin de faire évoluer la relation. L'étude des situations de conflit devient capitale, puisque leur situation de cas extrême fait ressortir un certain nombre d'éléments saillants. Selon nous, l'étude de ces conflits interactionnels passe notamment par une analyse de la sémantisation des individus après l'émergence du conflit, qui ouvre sur une étude a posteriori de la situation d'affrontement; nous préconisons l'enquête linguistique, non pas parce que la langue constitue le seul et unique moyen de sémantisation dans la communication (il est de fait que l'importance des échanges paraverbaux et non verbaux reste à souligner), mais parce qu'elle offre un ensemble d'expressions et de locutions susceptibles de retranscrire de manière plus ou moins fidèle et précise les ressentis et les besoins des interactants entrés en conflit. Pour ce faire, toujours dans un souci d'ordre pragmatique et une préservation des effets de l'environnement sur les interactants, nous préconisons l'utilisation de l'ESM ou " experiential sampling method " $^{39}$ ", grâce auquel les individus eux-mêmes tiennent un carnet ou un journal où ceux-ci décrivent leurs sentiments ou leurs pensées suite à un désaccord ou un conflit. La méthode ESM est actuel-

39 Joel Hektner, Jennifer Schmidt et Mihaly Csikszentmihalyi, Experience Sampling Method: Measuring the Quality of Everyday Life, Tousand Oaks, Sage, 2007. 
lement préconisée par de récents travaux en sociolinguistique ${ }^{40}$ mais également par les anthropologues spécialistes des questions identitaires ${ }^{41}$. Ces propositions approfondissent l'étude des interactions humaines à travers les nœuds sémantiques, qui autorisent ensuite la transmission et l'interprétation de l'information dans le système, ainsi que la maintenance ou la modification de la relation dans un contexte donné, comme le précise Peter Mühlhäusler dans son approche écologique des interactions :

From an ecological perspective meaning arises through the involvement of speakers with other speakers within a shared context of situation, and is shaped by their expectations, and their understandings of the world. Very importantly, meaning needs to be understood as part of ongoing discourses, not as located in decontextualized chunks of language (...). Language, because it depends on functional links with the outside world and because it is an inextricable part thereof, is thus an ecological phenomenon ${ }^{42}$.

D’après les travaux de Mühlhäusler, le contexte en tant qu'environnement interactionnel ne saurait être séparé de l'étude des occurrences linguistiques générée par l'ESM. Le sens émanant de ces deux éléments est observable comme une émergence situationnelle définie par un certain nombre de paramètres : un individu peut ainsi détester un certain trait de caractère (la cupidité, par exemple) et le trouver tout à fait rédhibitoire lorsqu'il s'agit de lier de nouvelles relations, mais accepter ce trait

40 Patricia Lamarre et Stéphanie Lamarre, «Montreal "on the move”: pour une approche ethnographique non-statique des pratiques langagières des jeunes multilingues ", dans Thierry Bulot (dir.), Formes et normes sociolinguistiques. Ségrégations et discriminations urbaines, Paris, L'Harmattan, 2009, p. 105-134.

41 Peter Burke et Jan Stets, Identity Theory, Oxford, Oxford University Press, 2009.

42 Peter Mühlhäusler, Language of Environment, Environment of Language : A Course in Ecolinguistics, London, Battlebridge, 2003, p. 9; "Dans une perspective écologique, la signification émerge à travers l'implication de locuteurs avec d'autres locuteurs, dans une situation de contexte partagée; cette signification est dessinée par leurs attentes et leurs façons de comprendre le monde. Il est important de concevoir la signification comme une partie de discours perpétuellement en cours, et non comme des éléments localisés dans des morceaux de langage décontextualisés (...). Le langage est donc un phénomène écologique, dans la mesure où il dépend de liens fonctionnels avec le monde extérieur et où il en est une partie inextricable » (notre traduction). 
de caractère en fonction des environnements interactionnels dans lesquels il se retrouve (s'il doit par exemple faire affaire avec un collaborateur ou dialoguer avec un membre de sa famille, etc.). Cela signifie qu'en fonction des contextes, nous activons et combinons un certain nombre d'éléments qui laissent émerger une interprétation de la situation en cours afin de s'y adapter de façon adéquate, et ce dans une apparente perspective de contradiction. Il est donc hasardeux de qualifier les individus en fonction d'un certain nombre de traits; en fonction du contexte, les interactants sont poussés à réactiver tel ou tel trait, comme exigé par les contraintes environnementales et sociales dans lesquelles ils doivent évoluer. De fait, les choix auxquels nous sommes soumis lors d'interactions n'obéissent pas à une linéarité engendrée par un certain nombre de données individuelle et interprétatives stables, mais s'expriment plutôt par un certain nombre d'inférences et d'implémentations d'interprétations d'informations ou ensembles d'information ${ }^{43}$ afin de résoudre les paradoxes causés par les environnements sociaux. Ces paradoxes sont descriptibles, dans la mesure où le modèle connexionniste prend en considération non pas le poids des informations traitées par l'interactant, mais le poids des connexions effectuées entre les différentes informations, comme le rappelle Gary Marcus :

Any given network architecture can represent a variety of different relationships between the input and output nodes, depending on the weights of the connections between units ${ }^{44}$.

La valeur que nous accordons aux nœuds sémantiques, ainsi que leur impact sur l'ensemble de notre appréciation de la situation interactionnelle sont des éléments essentiels. Ainsi, l'application de ces recherches fondamentale apporte une meilleure compréhension de cet animal étrange qu'est l'homme en

43 Jeffrey L. Elman, Elizabeth A Bates, Mark H. Johnson, Annette KarmiloffSmith, Domenico Parisi, Kim Plunkett, Rethinking Innateness. A Connectionist Perspective on Development, Cambridge, MIT Press, 1998, p. 63-66.

44 Gary Marcus, The Algebraic Mind. Integrating Connectionism and Cognitive Science, Cambridge, MIT Press, 2001, p. 11; " Tout réseau d'architecture donné peut représenter une variété de relations différentes entre les nœuds d'entrée et de sortie, en fonction du poids des connexions entre les unités" (notre traduction). 
interaction, avec ses choix, ses doutes, ses contradictions et ses formidables capacités d'adaptation, capable d'organiser et d'ordonner de grandes quantités d'information afin de pouvoir les activer en fonction des situations.

\section{Bibliographie}

Atlan, Henri, Entre le cristal et la fumée, Paris, Seuil, 1972.

Auchlin, Antoine, "Compétence discursive et co-occurrence d'affects : "blends expérientiels" ou (con)fusion d'émotions ", dans Jean-Marc Colletta et Anna Tcherkassof (dir.), Les Émotions. Cognition, langage et développement, Sprimont, Mardaga, 2003, p. 137-152.

Baars, Benjamin, In the Theater of Consciousness. The Workspace of the Mind, Oxford, Oxford University Press, 1997.Bange, Pierre, Analyse conversationnelle et théorie de l'action, Paris, Hatier/Didier, 1992.

Bateson, Gregory, Steps to an Ecology of Mind: Collected Essays in Anthropology, Psychiatry, Evolution and Epistemology, Chicago, The University of Chicago Press, 1972.

Bechtel, William et Adele Abrahamsen, Le Connexionnisme et l'esprit. Introduction au traitement parallèle par réseaux, Paris, La Découverte, 1993.

Burke, Peter et Jan Stets, Identity Theory, Oxford, Oxford University Press, 2009.

Camilleri, Carmel et Margalit Cohen-Emerique, Chocs de cultures : concepts et enjeux pratiques de l'interculturel, Paris, L'Harmattan, 1989.

Castella, Paul, La Différence en plus, Paris, L'Harmattan, 2005.

Costa de Beauregard, Olivier, Le Second principe de la science du temps, Paris, Seuil, 1963.

De Rosnay, Joël, Le Macroscope, Paris, Seuil, 1975.Dennett, Daniel, La conscience expliquée, Paris, Odile Jacob, 1993.

Dennet, Daniel, La Conscience expliquée, Paris, Odile Jacob, 1993.

Elman, Jeffrey L., Elizabeth A. Bates, Mark H. Johnson, Annette KarmiloffSmith, Domenico Parisi, Kim Plunkett, Rethinking Innateness. A Connectionist Perspective on Development, Cambridge, MIT Press, 1998.

Enfield, Nick et Stephen Levinson, "On the Human "Interaction 
Engine" ", dans Nick Enfield et Stephen Levinson (dir.), Roots of Human Sociality, New York, Berg, 2006, p. 39-69.

Frijda, Nico, "Passions : l'émotion comme motivation ", dans Jean-Marc Colletta et Anna Tcherkassof (dir.), Les Émotions. Cognition, langage et développement, Sprimont, Mardaga, 2003, p. 15-32.

Gehlen, Arnold, Anthropologie et psychologie sociale, Paris, Presses universitaires de France, 1990.

Girard, René, Des choses cachées depuis la fondation du monde, Paris, Grasset, 1978.

Goodwin, Charles et Alessandro Duranti, Rethinking Context. Language as an Interactive Phenomenon, Cambridge, Cambridge University Press, 1992.

Grice, Paul, "Logique et conversation ", Communications, n 30, 1979, p. $57-72$.

Guérin, Christophe, "Du positionnement à la posture : l'émergence ", Mémoire, Angers, École française de Yoga de l'Ouest, 2003.

Hektner, Joel, Jennifer Schmidt et Mihaly Csikszentmihalyi, Experience Sampling Method: Measuring the Quality of Everyday Life, Tousand Oaks, Sage, 2007.

Ionescu, Serban, Claude Lhote et Marie-Madeleine Jacquet, Les Mécanismes de défense, Paris, Armand Colin, 2005.

Kallmeyer, Werner, "L'Analyse de l'action dans la conversation ", dans Pierre Bange (dir.), L'Analyse des interactions verbales. La dame de Caluire. Une consultation, Bern, Peter Lang, 1987, p. 189-251.

Kamenarovic, Ivan, Le Conflit, Paris, Le Cerf, 2001.

Kerbrat-Orecchioni, Catherine, La Conversation, Paris, Seuil, 1996.

Kirouac, Gilles, Cognition et émotions, Coimbra, Imprensa da Universidade de Coimbra, 2004.

Lamarre, Patricia et Stéphanie Lamarre, " Montreal on the move": pour une approche ethnographique non-statique des pratiques langagières des jeunes multilingues ", dans Thierry Bulot (dir.), Formes et normes sociolinguistiques. Ségrégations et discriminations urbaines, Paris, L'Harmattan, 2009, p. 105-134.Laks, Bernard, Langage et cognition, Paris, Hermès, 1996.

Lerbet-Séréni , Frédérique, La Relation duale, Paris, L'Harmattan, 1994.

Lewis, David, Convention. A Philosophical Study, Harvard, Harvard University Press, 1969.

Locher, Miriam, Power and Politeness in Action, Berlin, Mouton de Gruyter, 2004.Malewska-Peyre, Hanna, "Problèmes d'identité des adolescents enfants de migrants et travail social ", dans Carmel Camilleri et 
Margalit Cohen-Emerique (dir.), Chocs de cultures : concepts et enjeux pratiques de l'interculturel, Paris, L'Harmattan, 1989, p. 117-134.

Marcus, Gary, The Algebraic Mind. Integrating Connectionism and Cognitive Science, Cambridge, MIT Press, 2001.

Meunier, Jean-Pierre, Approches systémiques de la communication, Bruxelles, De Boeck, 2003.

Monroy, Michel et Anne Fournier, Figures du conflit, Paris, Presses universitaires de France, 1998.

Morin, Edgar, La Méthode, tome 1, La nature de la nature, Paris, Seuil, 1977.Moser, Gabriel, Les relations interpersonnelles, Paris, Presses universitaires de France, 1994.

Mühlhäusler, Peter, Language of Environment, Environment of Language : A Course in Ecolinguistics, London, Battlebridge, 2003.Paulhan, Isabelle et Marc Bourgeois, Stress et coping. Les stratégies d'ajustement à l'adversité, Paris, Presses universitaires de France, 1995.

Prigogine, Ilya et Isabelle Stengers, La Nouvelle alliance, Paris, Gallimard, 1979.Reyna, Stephen, Connections. Brain, Mind, and Culture in Social Anthropology, London, Routledge, 2002.

Rimé, Bernard, Marie-Pascale Noël et Pierre Philippot, «Épisode émotionnel, réminiscences mentales et réminiscences sociales ", Les Cahiers internationaux de psychologie sociale, $\mathrm{n}^{\circ} 11,1991$, p. 93-104.

Varela, Francisco, Eleanor Rosch et Evan Thompson, L'Inscription corporelle de l'esprit. Sciences cognitives et expérience humaine, Paris, Seuil, 1993.

Varela, Francisco, Ethical Know-How: Action, Wisdom and Cognition, Stanford, Stanford University Press, 1999.

Von Bertalanffy, Ludwig, Théorie générale des systèmes, Paris, Dunod, 1973.

Wagener, Albin, "On Intercultural Disagreement : Interaction and Inertia ", dans Reyes Gomez Moron, Manuel Padilla Cruz, Lucia Fernandez Amaya et Maria de la O Hernandez Lopez (dir.), Pragmatics Applied to Language Teaching and Learning, Newcastle upon Tyne, Cambridge Scholars Publishing, 2009, p. 256-279.

Wagener, Albin, «L'Émotion dans l'émergence du désaccord interculturel », dans Béatrice Pothier (dir.), Langue, langage et interactions culturelles, Paris, L'Harmattan, 2009, p. 133-153.

Wagener, Albin, "Cooperative Disagreement: Towards a Definition of Discordant Interactions », dans Iwona Witczak-Plisiecka (dir.), Pragmatic Perspectives on Language and Linguistics, vol. 1, Speech Actions in Theory and Applied Studies, Newcastle upon Tyne, Cambridge Scholars Publishing, 2010, p. 171-197. 